\title{
THE DEEP2 GALAXY REDSHIFT SURVEY: REDSHIFT IDENTIFICATION OF SINGLE-LINE EMISSION GALAXIES
}

\author{
Evan N. Kirby, Puragra Guhathakurta, S. M. Faber, and David C. Koo \\ University of California Observatories/Lick Observatory, Department of Astronomy and Astrophysics, \\ University of California, Santa Cruz, CA 95064; ekirby@ucolick.org \\ BenJAMIN J. WeINER \\ Steward Observatory, University of Arizona, Tucson, AZ 85721 \\ AND \\ Michael C. Cooper \\ Department of Astronomy, University of California, Berkeley, CA 94720 \\ Received 2006 December 28; accepted 2007 January 29
}

\begin{abstract}
We present two methods for determining spectroscopic redshifts of galaxies in the DEEP2 survey which display only one identifiable feature, an emission line, in the observed spectrum ("single-line galaxies"). First, we assume each single line is one of the four brightest lines accessible to DEEP2: $\mathrm{H} \alpha$, [O III] $\lambda 5007, \mathrm{H} \beta$, or [O II] $\lambda 3727$. Then, we supplement spectral information with $B R I$ photometry. The first method, parameter-space proximity (PSP), calculates the distance of a single-line galaxy to galaxies of known redshift in $(B-R),(R-I), R, \lambda_{\text {observed }}$ parameter space. The second method is an artificial neural network (ANN). Prior information, such as allowable line widths and ratios, rules out one or more of the four lines for some galaxies in both methods. Based on analyses of evaluation sets, both methods are nearly perfect at identifying blended [O II] doublets. Of the lines identified as $\mathrm{H} \alpha$ in the PSP and ANN methods, $91.4 \%$ and $94.2 \%$, respectively, are accurate. Although the methods are not this accurate at discriminating between [O $\mathrm{OII}]$ and $\mathrm{H} \beta$, they can identify a single line as one of the two, and the ANN method in particular unambiguously identifies many [O III] lines. From a sample of 640 single-line spectra, the methods determine the identities of $401(62.7 \%)$ and $472(73.8 \%)$ single lines, respectively, at accuracies similar to those found in the evaluation sets.
\end{abstract}

Subject headings: galaxies: distances and redshifts - line: identification

Online material: color figures

\section{INTRODUCTION}

Photometric redshifts (photo-zs) save astronomers from expensive spectroscopy by determining redshifts from efficient broadband photometry. However, photo- $z$ precision cannot compare to spectroscopic redshift precision. Way \& Srivastava (2006) compare five state-of-the-art methods to determine redshifts from Sloan Digital Sky Survey (SDSS) photometry. Neural networks, which are nonlinear regression tools, perform the best, but the rms error in the photo-zs is $\delta z /(1+z) \sim 0.02$ at best. This precision is sufficient to study large-scale structure and some forms of redshift evolution, but not local environments (Cooper et al. 2005), kinematic pairs, or the low-redshift luminosity function, where precise luminosities require precise redshifts. At a spectral resolution $R=5000$, the spectroscopic redshifts in the Deep Extragalactic Evolutionary Probe (DEEP2) Galaxy Redshift Survey (Davis et al. 2003) are many times more precise. Repeat observations show redshift errors of $\delta z \sim 10^{-4}$, or velocity errors of $\delta v \sim 30 \mathrm{~km} \mathrm{~s}^{-1}$.

The expense of time-intensive spectroscopy demands a high rate of successfully determined redshifts. Nonetheless, some spectra fail at providing redshifts. One of the most common redshift failures is the presence of only one emission line. Ordinarily, recognizable patterns of emission or absorption lines uniquely determine the lines' identities and hence rest wavelengths. Spectroscopically precise observed wavelengths then give highly precise redshifts. An isolated emission line forms no recognizable pattern to reveal its identity and rest wavelength.
Recovering failed spectroscopic redshifts with broadband photometry has hardly been explored in the literature. Cohen et al. (1999) assume all single emission lines in the Caltech Faint Galaxy Redshift Survey (CFGRS) are [O II] $\lambda 3727$ by arguing that any other line except Ly $\alpha$ would be accompanied by other emission lines. Lilly et al. (1995) have identified single emission lines in Canada-France Redshift Survey (CFRS) spectra based on the slope of the continuum surrounding the line. (Unlike DEEP2, both CFGRS and CFRS did not have the spectroscopic resolution to resolve the [ $\left.\mathrm{O}_{\mathrm{II}}\right]$ doublet, requiring them to make assumptions about the identities of single lines.)

In this paper, we also rely on the shape of the continuum, but we will show that broadband $B R I$ colors can determine the identity of single emission lines accurately, even for galaxies with no visible spectral continuum. The problem of an isolated emission line afflicts $\sim 1.5 \%$ of DEEP 2 targets, but the problem is more prevalent for serendipitously detected galaxies ("serendips") that share a slit with target galaxies through a fortuitous position on the sky. Recovery of redshifts from serendip spectra is important because serendips form an unbiased sample of galaxy spectra.

We explore the problem through two methods. The parameterspace proximity (PSP) method identifies the redshift of a singleline galaxy by comparing its photometric magnitude and colors with those of galaxies with known spectroscopic redshifts. The artificial neural network (ANN) method employs the ANN machine-learning algorithm, which learns the functional relationship between any number of dependent and independent variables. ANNs can determine photometric redshifts from relevant 
observable quantities such as colors, apparent magnitudes, and angular sizes. Here, we use apparent magnitudes (from which the ANN determines colors) and observed wavelengths of the single emission lines.

This paper is organized as follows: $\S 2$ describes the DEEP2 survey and how single emission lines arise in its spectra; $\S 3$ describes the two methods for determining the identities of these single lines; $\S 4$ examines the accuracy of each method; $\S 5$ presents the results of applying the methods to single emission lines; and $\S 6$ summarizes our work and discusses our future plans.

\section{DATA}

\subsection{DEEP2 Survey}

The DEEP2 Survey (outlined by Davis et al. 2003) combines $B R I$ photometry in four fields (described by Coil et al. 2004) from the CFHT $12 \mathrm{~K} \times 8 \mathrm{~K}$ mosaic camera and spectroscopy (described by J. A. Newman et al. 2007, in preparation) from the DEIMOS spectrograph (Faber et al. 2003) on the Keck II telescope. Davis et al. (2005) detail the DEEP2 target selection, based on the three-filter $(B, R, I)$ photometry. A color-color cut is applied to spectroscopy candidates in three of the four $120 \mathrm{arcmin}^{2}$ DEEP2 fields to reduce as much as possible the number of spectra of $z<0.7$ galaxies. The remaining field (field 1) has no such color-color selection. An apparent magnitude cut of $R_{\mathrm{AB}}<24.1$ is applied to all fields. Furthermore, each spectroscopy target must be detected in $B, R$, and $I$. The spec2d and spec1d software packages, written by the DEEP2 team, accomplish the DEIMOS spectroscopy reduction, including sky subtraction and an instrumental throughput correction to preserve actual line strengths.

Field 1 is unique in other ways as well. It overlaps the extended Groth strip (EGS), which many different teams and instruments observe heavily. The wealth of photometry for the DEEP2 targets in field 1 permits very good photo-z estimates (J.-S. Huang et al. 2007 , in preparation). We plan to make use of the additional EGS data in future work. However, in this paper we use only the $B R I$ photometry in field 1 , and we treat it no differently from fields 2-4.

Astronomers from the DEEP2 team visually inspect every spectrum using the zspec script from the spec1d software package, designed by DEEP 2 members. They assign spectra with at least two strong identifiable lines - such as an unblended [O II] doublet - a redshift quality code $Q=4$. Spectra with one strong line and at least one weaker line receive $Q=3$. The entire survey contains 49,059 spectra. Of them, 27,460 and 5829 are $Q=4$ and 3 , respectively. The $Q=2$ category encompasses all failed redshifts that may be recovered with additional effort. The inspectors note the reason for all failures in this category, including the absence of all but one line.

\subsection{Single-Emission-Line Galaxies}

The spectral resolution of a DEEP2 spectrum is $1.4 \AA$ FWHM, and the typical spectral range is about $6600-9200 \AA$. Therefore, few redshifts permit both $\mathrm{H} \alpha \lambda 6563$ and [O III] $\lambda 5007$ or $\mathrm{H} \beta$ $\lambda 4861$ to fall on the same spectrum. Similarly, few redshifts permit both [O III] or $\mathrm{H} \beta$ and [O $\mathrm{II}]$ 23727 . Consequently, most DEEP2 galaxies which are too faint to have a weaker emission line or a continuum with noticeable absorption lines display only one visible feature: $\mathrm{H} \alpha$, [O $\mathrm{III}], \mathrm{H} \beta$, or [O $\mathrm{II}]$ in emission. This feature is often the [O II] doublet, which is easily identifiable by its invariable $220 \mathrm{~km} \mathrm{~s}^{-1}$ peak separation as long as the galaxy's internal gas velocity dispersion does not broaden and blend both peaks. However, the feature may be a truly isolated single line, in which case the spectrum cannot yield a unique redshift. Lines may appear to be orphans if the signal-to-noise ratio is low enough to permit only the brightest line to be seen. Other weak lines may be lost in the noise of night-sky lines, even with good sky subtraction. Finally, a gap of $\sim 5 \AA$ separates the red and blue CCDs in DEIMOS, and any line in a pair of otherwise visible lines that falls completely in the gap will orphan its partner.

We select all 984 redshift failures that result from a single emission line. After an additional visual inspection to remove spectra with marginally detected lines, spectra mistakenly marked as having a single line, and spectra without any visible emission lines, our sample contains 640 single-line emission galaxies. We identify the pixel within the line that contains the most counts in the spectrum smoothed through a Gaussian window function with $\sigma=4$ pixels $=1.3 \AA$ and inverse variance weighting. We designate that pixel's observed wavelength as the wavelength of the single line. Finally, we note whether the line is broad enough to be a blended [O II] doublet. For the purposes of identifying lines, it is not necessary to be more precise in determining the observed wavelength of the [O II] doublet than selecting the pixel with the most counts.

It is worth mentioning that the line identification methods presented here will fail at identifying the redshifts of composite galaxies, such as those blended together through lensing or lineof-sight coincidence. We assume that the broadband colors and magnitudes associated with all of the lines identified come from a single galaxy, and that composite colors and magnitudes will be distinct enough in parameter space that their associated single lines will not receive a conclusive identification.

\section{METHODS}

The bright lines most often visible in DEEP2 spectra are $\mathrm{H} \alpha$ $\lambda 6563$, [O III] $\lambda 5007, \mathrm{H} \beta \lambda 4861$, and the [O II] $\lambda \lambda 3726,3729$ doublet. If any other line is visible, then one of these four is almost always visible as well. The $B$-band detection requirement eliminates the possibility of Ly $\alpha$. On rare occasions, bright skylines or the DEIMOS CCD gap may hide one of these four lines, leaving only one other dimmer line, such as [O III $] \lambda 4959$ or $\mathrm{H} \gamma$ $\lambda 4341$, visible. For this paper, we assume that any single emission line is one of the four bright lines.

If a line is observed at $\lambda_{0}$, then we assume the galaxy's redshift is one of $z_{\mathrm{H} \alpha}=\lambda_{0} / 6563 \AA-1, z_{\mathrm{O}}=\lambda_{0} / 5007 \AA-1, z_{\mathrm{H} \beta}=$ $\lambda_{0} / 4861 \AA-1$, or $z_{\text {OII }}=\lambda_{0} / 3727 \AA-1$. We employ two independent methods, described below, to assign probabilities to the four possible line identities or redshifts: $P_{\mathrm{H} \alpha}, P_{\mathrm{O}}, P_{\mathrm{H} \beta}$, and $P_{\mathrm{O}}$ I. In addition, we set to zero the probabilities of lines that satisfy the following conditions:

1. If the line is observed bluer than $0.98 \lambda_{\mathrm{H} \alpha}=6431.5 \AA$, then $P_{\mathrm{H} \alpha}=0$. In other words, we do not permit blueshifts $z<-0.02$.

2. Of the $Q=3$ and $Q=4$ DEEP2 galaxies at redshifts where both $\mathrm{H} \alpha$ and $\mathrm{H} \beta$ are accessible, $80 \%$ exhibit a Balmer decrement of $2.6<\mathrm{H} \alpha / \mathrm{H} \beta<7.7$, where the unit is spectral counts at the line peak. Therefore, if the line is assumed to be $\mathrm{H} \alpha$, and $\mathrm{H} \beta$ also falls within the DEIMOS slit's spectral range, then $P_{\mathrm{H} \alpha}=0$ if the smoothed spectral counts at the location of the single line are less than 2.6 or greater than 7.7 times the counts at the location of $\mathrm{H} \beta$ within the errors of the photon-counting statistics.

3. Similarly, $80 \%$ of $Q=3$ and $Q=4$ DEEP2 spectra with both $[\mathrm{O}$ III $]$ lines exhibit an $[\mathrm{O}$ III] doublet ratio of $1.6<[\mathrm{O}$ III $]$ $\lambda 5007 /[\mathrm{O}$ III $] \lambda 4959<5.0$. Therefore, if the line is assumed to be [O III] $\lambda 5007$ and [O III] $\lambda 4959$ should also be visible, then 
$P_{\mathrm{O} \text { III }}=0$ if the counts at the location of $[\mathrm{O}$ III $] \lambda 5007$ are less than 1.6 or greater than 5.0 times the counts at the location of [O III] $\lambda 4959$ within the errors of the photon-counting statistics. The true physical ratio is always [O III $] \lambda 5007 /[\mathrm{O}$ III $] \lambda 4959=3$ (Osterbrock \& Ferland 2006).

4. In $80 \%$ of $Q=3$ and $Q=4$ DEEP 2 spectra with both [O III] $\lambda 5007$ and $\mathrm{H} \beta, 0.49<[\mathrm{O}$ III $] / \mathrm{H} \beta<4.2$. Therefore, $P_{\mathrm{O}_{\text {III }}}=0$ if $[\mathrm{O} \mathrm{III}] / \mathrm{H} \beta>4.2$ within the errors of the photon-counting statistics.

5. Following condition $2, P_{\mathrm{H} \beta}=0$ if $\mathrm{H} \beta / \mathrm{H} \alpha>0.34$ within the errors of the photon-counting statistics.

6. Following condition $4, P_{\mathrm{H} \beta}=0$ if $\mathrm{H} \beta /[\mathrm{O}$ III $]>1.6$ within the errors of the photon-counting statistics.

7. If the line drops to no visible counts within a window smaller than $220 \mathrm{~km} \mathrm{~s}^{-1}$, the velocity separation of the [O II] doublet, then $P_{\mathrm{O} \text { II }}=0$ because the line cannot be dispersionblended [O II].

Conditions $2-6$ rely on an $80 \%$ confidence interval, which may seem strict, but the large majority of the typically low signal-tonoise single lines pass these tests by virtue of their large photoncounting errors. After all priors have been applied, we normalize the remaining probabilities $P$ such that their sum is unity.

In the language of photo-zs, both of the following techniques are "training set" methods, or empirical calibrations. Training methods are immune to improper modeling and even incorrect photometric zero-point offsets. One weakness of training methods is that they require large training sets for their accuracy and precision to compare to that of modeling methods.

Another weakness is that they assume that the samplepopulation properties are similar to the training-set properties. For example, if single-line galaxies preferentially have lower metallicity and bluer colors than training-set galaxies, then the methods described here will search a skewed region of parameter space. Furthermore, a larger fraction of single-line galaxies show little or no continuum than training-set galaxies, which may skew colors. For this paper, we assume that the photometric properties of single-line galaxies are a subset of the photometric properties of training-set galaxies, meaning that the parameter space around each single-line galaxy is well populated by training-set galaxies at similar redshifts.

\subsection{Known-Redshift Sets}

Both methods which assign probabilities to each of the four major lines require sets of galaxies with well-measured redshifts. The known-redshift set consists of DEEP2 targets with quality $Q=3$ or 4 spectroscopic redshifts and with at least one emission line detected at least $5 \sigma$ above the noise. There are 20,676 such galaxies. We identify the emission line containing the pixel with the maximum counts in the spectrum smoothed in the same manner as the single-line galaxy spectra in $\S 2.2$. For the remainder of the training process, we treat the spectrum as containing only that single line, but the redshift - and hence the identity of the single line - is known.

Inaccurate redshifts in the known-redshift set of course lead to inaccurate training and spurious line identification. Repeat observations have shown that fewer than $4.5 \%$ of the $2061 Q=3$ redshifts and fewer than $0.5 \%$ of the $18,615 Q=4$ redshifts are incorrect. Therefore, the known-redshift set is less than $2 \%$ contaminated by incorrect redshifts.

Both of the following methods assume that the training-set galaxies populate the same observable parameter space as true single-line galaxies. The different target selection functions between field 1 (6532 galaxies) and fields 2-4 (14,144 galaxies) make this point especially important. The underrepresentation of $z<0.7$ galaxies, which are present almost exclusively in field 1 , decreases the certainty at which $z<0.7$ galaxies may be identified. However, as long as $z<0.7$ single-line galaxies populate a region of parameter space distinct from $z>0.7$ galaxies, and as long as $z<0.7$ training-set galaxies also populate that region, the following algorithms should not mistake low-redshift galaxies for high-redshift ones. Therefore, we combine galaxies from all fields into one training set. We have also analyzed both methods with field segregation. We tested field 1 galaxies using a training set with only field 1 galaxies, and we tested fields $2-$ 4 galaxies using a training set with only fields $2-4$ galaxies. The accuracy was statistically indifferent from using a unified training set, but the number of conclusively identified galaxies decreased slightly.

\subsection{Parameter-Space Proximity Method}

We randomly divide the known-redshift set into training and evaluation subsets. The latter plays no role in the training, and we invoke it only in $\S 4$. A larger training set yields higher precision whereas a larger evaluation set provides more confident tests of the method. We find that diverting $20 \%$ of the knownredshift set into the evaluation set, leaving $80 \%$ for the training set, gives a significant, untouched sample by which to judge performance without significantly affecting precision.

The survey's three available broadband filter measurements, $B, R$, and $I$, permit photo- $z$ measurements. Because emissionline galaxies in different redshift ranges form different loci in $(B-R),(R-I), R, \lambda_{\text {observed }}$ space, a single galaxy's position in that plane gives a guess at its redshift. The two colors alone provide enough information to determine some line identities, but adding $R$ apparent magnitude increases the number of identifications by $\sim 15 \%$. Observed wavelength also provides discriminatory power because both the single line's observed wavelength and the galaxy's broadband colors are functions of redshift.

We assign an identification confidence parameter $P_{X}^{j}$ for each single-line galaxy, represented by index $j . X$ represents one of the bright lines. Roughly, the confidence parameter is a measure of distances from the point of the sample galaxy to each point in the training set in parameter space. More or closer points give a higher $P_{X}^{j}$, and fewer or farther points give a lower $P_{X}^{j}$. See Figure 1 for a simplified representation of this method.

More precisely, every point in the training set is assigned a four-dimensional Gaussian. Each of the four axes corresponds to

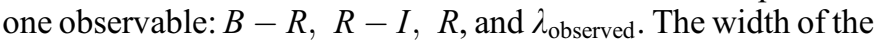
Gaussian for each of the three color and magnitude observables is given by the sum in quadrature of the photometric error for the training-set galaxy $i$ and the photometric error of the single-line galaxy $j$. The wavelength Gaussian is unique because it distinguishes between the four line identities. Each galaxy in the training set has four wavelength Gaussians, each corresponding to one of the four possible line identities, represented by $X$. The wavelength at the peak of Gaussian $X$ is $(1+z) \lambda_{X}$, where $z$ is the known redshift of the training-set galaxy and $\lambda_{X}$ is the rest wavelength of one of the four lines. The width of this Gaussian is the sum in quadrature of $\delta_{\lambda}=1000 \delta z \lambda_{X}$, where $\delta z$ is the error in the redshift from the spec1d spectral template cross-correlation, and $106 \AA$, the median value of $\delta_{\lambda}$ for the evaluation set. The factor of 1000 is necessary to widen the Gaussians so that they actually overlap. It was chosen to optimize accuracy, but the results are very insensitive to the precise value. $P_{X}^{j}$ is the sum of the values of all of these Gaussians corresponding to line $X$ at the point where 

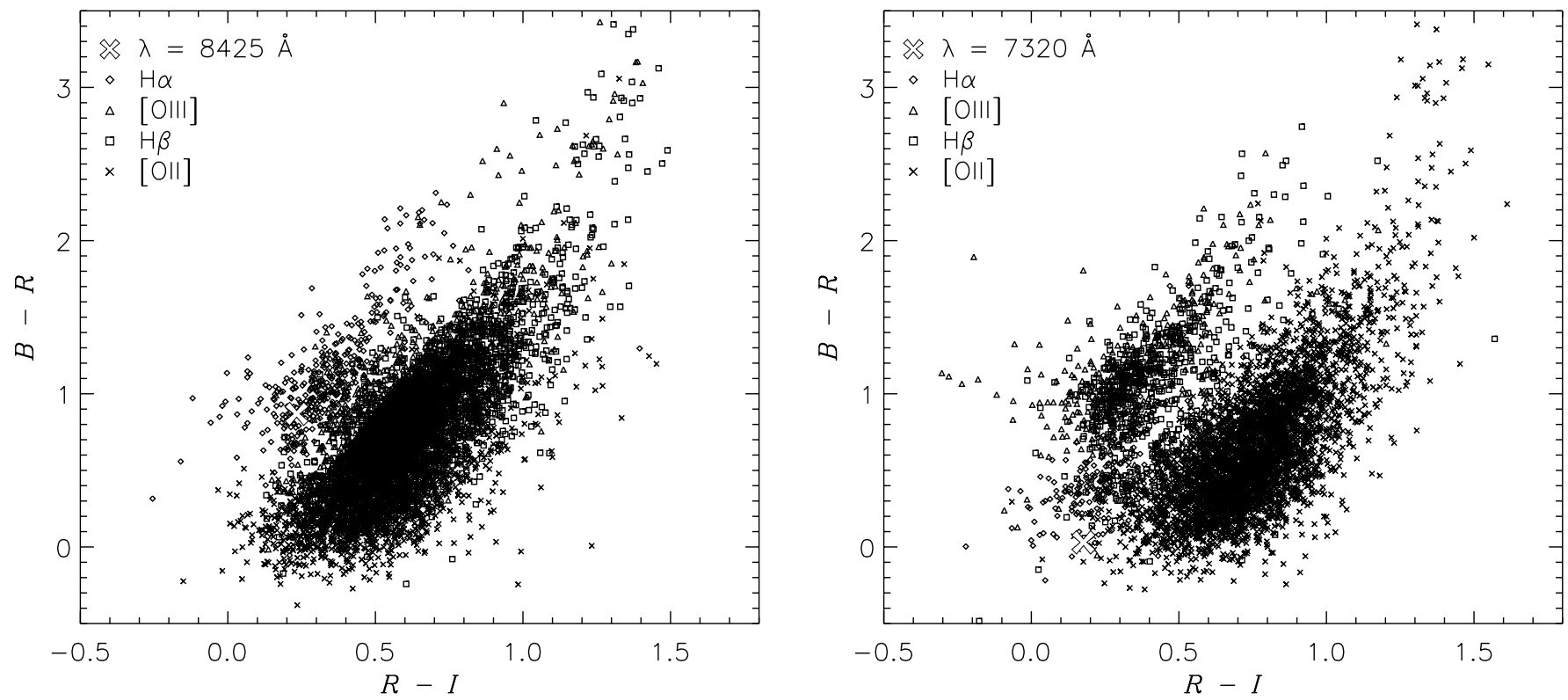

FIG. 1.-Left: Simplified representation of the PSP method, with the four-dimensional parameter space projected onto the $B R I$ color-color plane. Training-set galaxies are color-coded by the values of their wavelength Gaussians (see $\S 3.2$ ). Those galaxies whose $\mathrm{H} \alpha$ wavelength Gaussians exceed $5 \times 10^{-4}$ are red, and so on for the other three line identities. A galaxy with a single line observed at $8425 \AA$ (large cross) falls in the middle of a locus of red points. Therefore, this line is identified as H $\alpha$. In reality, each training-set point is a fixed-volume, four-dimensional Gaussian whose width is given by errors in observable quantities. The sum of all the Gaussians in a particular line category at the location of the single-line galaxy is the probability that the single line also falls into that category. The two suppressed axes are $R$ magnitude and observed wavelength. Right: The same figure for a galaxy with different colors and wavelength. In the absence of additional information, the identity of the single line cannot be determined because galaxies from multiple line identities populate the color-color space around the single-line galaxy. In both panels, the sharp color-color cut in fields $2-4$ ensures that only field 1 galaxies populate the upper left corner of the diagram. Note that $z_{\mathrm{H} \alpha}=0.28$ in the left panel and $z_{\mathrm{H} \alpha}=0.12$ in the right panel. The right panel has fewer $\mathrm{H} \alpha$ points because the differential volume of the universe is smaller at the second $z_{\mathrm{H} \alpha}$. [See the electronic edition of the Journal for a color version of this figure.]

the single-line galaxy $j$ lies in the four-dimensional parameter space. In symbols,

$$
\begin{aligned}
P_{X}^{j}= & \sum_{i} \frac{1}{\sigma_{B R} \sigma_{R I} \sigma_{R} \sigma_{\lambda}} \exp \left(-\frac{\left[(B-R)_{i}-(B-R)_{j}\right]^{2}}{2 \sigma_{B R}^{2}}\right. \\
& -\frac{\left[(R-I)_{i}-(R-I)_{j}\right]^{2}}{2 \sigma_{R I}^{2}}-\frac{\left(R_{i}-R_{j}\right)^{2}}{2 \sigma_{R}^{2}} \\
& \left.-\frac{\left[\left(1+z_{i}\right) \lambda_{X}-\lambda_{j}\right]^{2}}{2 \sigma_{\lambda}^{2}}\right) \\
\sigma_{B R}^{2} \equiv & \left(\delta B_{i}\right)^{2}+\left(\delta R_{i}\right)^{2}+\left(\delta B_{j}\right)^{2}+\left(\delta R_{j}\right)^{2} \\
\sigma_{R I}^{2} \equiv & \left(\delta R_{i}\right)^{2}+\left(\delta I_{i}\right)^{2}+\left(\delta R_{j}\right)^{2}+\left(\delta I_{j}\right)^{2} \\
\sigma_{R}^{2} \equiv & \left(\delta R_{i}\right)^{2}+\left(\delta R_{j}\right)^{2} \\
\sigma_{\lambda}^{2} \equiv & \left(1000 \delta z_{i} \lambda_{X}\right)^{2}+(106 \AA)^{2} .
\end{aligned}
$$

Although colors may be determined more precisely than equations (2) and (3) suggest, we use the error simply to broaden the Gaussians. In addition, the Gaussians of galaxies with larger photometric errors will contribute less to $P_{X}^{j}$ because they will have larger widths but the same volume.

The confidence parameters are subjected to the prior conditions described above and normalized such that $\sum_{X} P_{X}^{j}=1$. This normalization makes the Gaussian prefactor of $(2 \pi)^{-2}$ unnecessary. Thus, $P_{X}^{j}$ represents the probability that the identity of the single emission line in spectrum $j$ is $X$.

The criterion for a conclusive line identification is that $P_{X}^{j}$ for a certain line $X$ exceeds $\rho_{\mathrm{PSP}}$, a tunable parameter. A larger $\rho_{\mathrm{PSP}}$ will yield fewer conclusive line identifications, but a larger fraction of them will be correct. ${ }^{1}$

Because $[\mathrm{O}$ III] $\lambda 5007$ and $\mathrm{H} \beta \lambda 4861$ always fall so close to each other in wavelength, it is very difficult to discriminate between them. However, it is possible to rule out $\mathrm{H} \alpha$ and [O II] if $P_{\mathrm{O} \text { III }}^{j}+$ $P_{\mathrm{H} \beta}^{j}>\rho_{\mathrm{PSP}}$. In addition, conditions 3-6 described above may rule out either [O III] 25007 or $\mathrm{H} \beta$, leaving only one possibility.

\subsection{Artificial Neural Network Method}

An artificial neural network (ANN) can learn the functional relationship between certain elements in a data set and derived

${ }^{1}$ When referring to line identification, "accuracy" in this paper means identifying lines correctly. "Precision" means the confidence with which a line is identified, or its value of $P_{X}$. Therefore, increasing $\rho_{\text {PSP }}$ imposes a stricter condition on precision, thereby increasing accuracy.

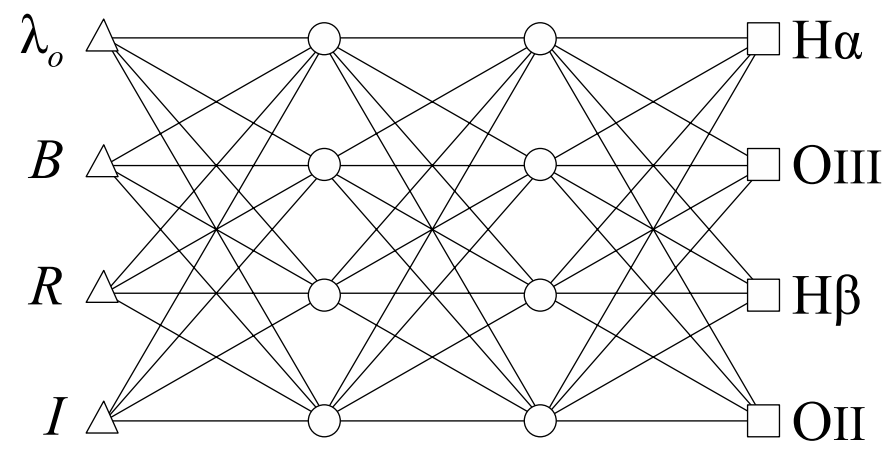

FIG. 2.-The 4:4:4:4 artificial neural network architecture used for determining the identities of single emission lines. Triangles represent the observed wavelength and three broadband filter inputs; squares represent the probabilities of each line identity; and circles represent hidden nodes. 
TABLE 1

Evaluation-Set Accuracy, All Fields

\begin{tabular}{|c|c|c|c|c|c|}
\hline \multirow[b]{2}{*}{ IDENTIFIED AS } & \multicolumn{4}{|c|}{ Actual Line Identity } & \multirow[b]{2}{*}{ Total } \\
\hline & $\mathrm{H} \alpha$ & [O III] & $\mathrm{H} \beta$ & [O II] & \\
\hline \multicolumn{6}{|c|}{ PSP Method } \\
\hline $\mathrm{H} \alpha$ & 201 & 15 & 2 & 2 & 220 \\
\hline$[\mathrm{O}$ III $] \ldots \ldots \ldots \ldots \ldots \ldots$ & 14 & 200 & 8 & 4 & 226 \\
\hline $\mathrm{H} \beta$ & 3 & 36 & 40 & 4 & 83 \\
\hline$[\mathrm{O}$ II $] \ldots \ldots \ldots \ldots \ldots$ & 0 & 0 & 0 & 1896 & 1896 \\
\hline$[\mathrm{O}$ III] or $\mathrm{H} \beta \ldots \ldots \ldots \ldots$ & 28 & 571 & 365 & 25 & 989 \\
\hline Inconclusive .................. & 63 & 79 & 9 & 567 & 718 \\
\hline Total ........................... & 309 & 901 & 424 & 2498 & 4132 \\
\hline \multicolumn{6}{|c|}{ ANN Method } \\
\hline ............ & 226 & 11 & 2 & 1 & 240 \\
\hline$[\mathrm{O}$ ІІІ $] \ldots \ldots \ldots \ldots \ldots \ldots$ & 14 & 545 & 32 & 5 & 596 \\
\hline 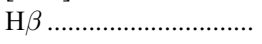 & 0 & 18 & 60 & 1 & 79 \\
\hline$[\mathrm{O}$ II] & 0 & 0 & 0 & 2184 & 2184 \\
\hline$[\mathrm{O}$ III] or $\mathrm{H} \beta \ldots \ldots \ldots \ldots$ & 8 & 217 & 316 & 16 & 557 \\
\hline Inconclusive .................... & 61 & 110 & 14 & 291 & 476 \\
\hline Total ……..................... & 309 & 901 & 424 & 2498 & 4132 \\
\hline
\end{tabular}

properties of the same set. The first major implementation of ANNs in astronomy was galaxy morphological classification (StorrieLombardi et al. 1992), but presently, the most common ANN implementation is photometric redshifts (e.g., Firth et al. 2003; Vanzella et al. 2004). ANNs are more precise than other machinelearning methods (e.g., trained decision tree classifiers; Suchkov et al. 2005) and model-independent, in contrast to template-fitting methods (e.g., Coe et al. 2006; Brodwin et al. 2006). Given a set of photometric data, such as broadband filter measurements, an ANN can estimate a redshift and the error on that redshift. This process requires a very large training set for accurate and precise results. Typically, a large known-redshift set with broadband data and spectroscopic redshifts is divided randomly into three independent sets: training, validation, and evaluation. (In this paper,

TABLE 2

Evaluation-Set Accuracy, Field 1

\begin{tabular}{|c|c|c|c|c|c|}
\hline \multirow[b]{2}{*}{ IDENTIFIED AS } & \multicolumn{4}{|c|}{ Actual Line IDENTity } & \multirow[b]{2}{*}{ Total } \\
\hline & $\mathrm{H} \alpha$ & [O III] & $\mathrm{H} \beta$ & [O II $]$ & \\
\hline \multicolumn{6}{|c|}{ PSP Method } \\
\hline $\mathrm{H} \alpha$ & 166 & 8 & 2 & 2 & 178 \\
\hline$[\mathrm{O}$ III] & 12 & 106 & 6 & 1 & 125 \\
\hline $\mathrm{H} \beta$ & 0 & 20 & 17 & 0 & 37 \\
\hline$[\mathrm{O}$ II] & 0 & 0 & 0 & 372 & 372 \\
\hline$[\mathrm{O}$ III] or $\mathrm{H} \beta \ldots \ldots \ldots \ldots \ldots . .$. & 19 & 223 & 133 & 7 & 382 \\
\hline Inconclusive ..................... & 50 & 62 & 4 & 96 & 212 \\
\hline Total ........................... & 247 & 419 & 162 & 478 & 1306 \\
\hline \multicolumn{6}{|c|}{ ANN Method } \\
\hline $\mathrm{H} \alpha$ & 179 & 5 & 0 & 1 & 185 \\
\hline$[\mathrm{O}$ ІІІ $] \ldots \ldots \ldots \ldots \ldots \ldots \ldots$ & 8 & 215 & 15 & 0 & 238 \\
\hline $\mathrm{H} \beta$ & 0 & 11 & 22 & 1 & 34 \\
\hline$[\mathrm{O}$ II] & 0 & 0 & 0 & 432 & 432 \\
\hline$[\mathrm{O}$ III] or $\mathrm{H} \beta \ldots \ldots \ldots \ldots \ldots . .$. & 5 & 105 & 113 & 3 & 226 \\
\hline 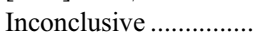 & 55 & 83 & 12 & 41 & 191 \\
\hline 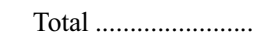 & 247 & 419 & 162 & 478 & 1306 \\
\hline
\end{tabular}

TABLE 3

Evaluation-Set Accuracy, Fields 2-4

\begin{tabular}{|c|c|c|c|c|c|}
\hline \multirow[b]{2}{*}{ IDENTIFIED AS } & \multicolumn{4}{|c|}{ Actual Line Identity } & \multirow[b]{2}{*}{ Total } \\
\hline & $\mathrm{H} \alpha$ & {$[\mathrm{O}$ III] } & $\mathrm{H} \beta$ & [O II] & \\
\hline \multicolumn{6}{|c|}{ PSP Method } \\
\hline $\mathrm{H} \alpha$. & 35 & 7 & 0 & 0 & 42 \\
\hline [O III] & 2 & 94 & 2 & 3 & 101 \\
\hline 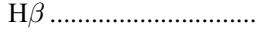 & 3 & 16 & 23 & 4 & 46 \\
\hline$[\mathrm{O}$ II] & 0 & 0 & 0 & 1524 & 1524 \\
\hline$[\mathrm{O}$ III] or $\mathrm{H} \beta \ldots \ldots \ldots \ldots$ & 9 & 348 & 232 & 18 & 607 \\
\hline Inconclusive .................. & 13 & 17 & 5 & 471 & 506 \\
\hline 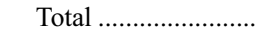 & 62 & 482 & 262 & 2020 & 2826 \\
\hline \multicolumn{6}{|c|}{ ANN Method } \\
\hline $\mathrm{H} \alpha$ & 40 & 5 & 2 & 0 & 47 \\
\hline$[\mathrm{O}$ III] $]$ & 6 & 334 & 16 & 3 & 359 \\
\hline $\mathrm{H} \beta$ & 0 & 11 & 41 & 0 & 52 \\
\hline$[\mathrm{O}$ II] $] \ldots \ldots \ldots \ldots \ldots \ldots \ldots \ldots \ldots \ldots \ldots \ldots \ldots$ & 0 & 0 & 0 & 1751 & 1751 \\
\hline$[\mathrm{O}$ III] or $\mathrm{H} \beta \ldots \ldots \ldots \ldots$ & 5 & 105 & 202 & 19 & 331 \\
\hline Inconclusive .................. & 11 & 27 & 1 & 247 & 286 \\
\hline Total ........................ & 62 & 482 & 262 & 2020 & 2826 \\
\hline
\end{tabular}

we divide the known-redshift set into $60 \%$ training, $20 \%$ validation, and $20 \%$ evaluation to balance the precision a large training set affords with the ability to test the ANN on an evaluation set.) The ANN learns the dependence of redshift on photometric observables from the training set and interactively verifies its accuracy with the validation set. After training and validation are complete, the ANN configuration is fixed and unchangeable. At this point, the evaluation set can test how well the ANN has been trained (see $\S 4$ ). Finally, the ANN may be applied to data without spectroscopic redshifts to obtain photometric redshifts with an accuracy comparable to that achieved with the evaluation set.

In determining single-emission-line identity, we employ an ANN with a four-component output, using the publicly available code ANNz (Collister \& Lahav 2004). Each output corresponds to the probability of a line identification. During training, the brightest line in the spectrum of a galaxy of known redshift is assigned a probability of 1 while the other probabilities are 0 . In addition to providing $B, R$, and $I$ measurements as input to the ANN, we also provide the wavelength of the single line. Although there is danger in using irrelevant data as input to an ANN, we justify the use of line wavelength by remarking that the redshift and hence $B R I$ magnitudes and colors of a galaxy would be different if the single line were $\mathrm{H} \alpha$ at observed wavelength $\lambda_{1}$ rather than a different $\lambda_{2}$. In full detail, the ANN architecture is $4: 4: 4: 4$, meaning 4 inputs, 4 outputs, and 2 hidden layers with 4 nodes

TABLE 4

Comparison between PSP and ANN Methods on Evaluation Set

\begin{tabular}{|c|c|c|c|c|c|c|c|}
\hline \multirow[b]{2}{*}{ PSP MEthOD } & \multicolumn{6}{|c|}{ ANN Method } & \multirow[b]{2}{*}{ TотAL } \\
\hline & $\mathrm{H} \alpha$ & [O III] & $\mathrm{H} \beta$ & [O II] & {$[\mathrm{O} \quad \mathrm{III}] / \mathrm{H} \beta$} & Inconclusive & \\
\hline$\ldots \ldots \ldots . .$. & 182 & 3 & 0 & 0 & 3 & 32 & 220 \\
\hline 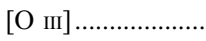 & 5 & 149 & 0 & 0 & 42 & 30 & 226 \\
\hline $\mathrm{H} \beta \ldots \ldots \ldots \ldots$ & 4 & 12 & 26 & 1 & 28 & 12 & 83 \\
\hline 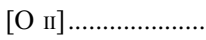 & 0 & 0 & 0 & 1839 & 1 & 56 & 1896 \\
\hline$[\mathrm{O}$ III] or $\mathrm{H} \beta \ldots \ldots \ldots$ & 11 & 411 & 52 & 1 & 464 & 50 & 989 \\
\hline Inconclusive .......... & 38 & 21 & 1 & 343 & 19 & 296 & 718 \\
\hline Total .................... & 240 & 596 & 79 & 2184 & 557 & 476 & 4132 \\
\hline
\end{tabular}



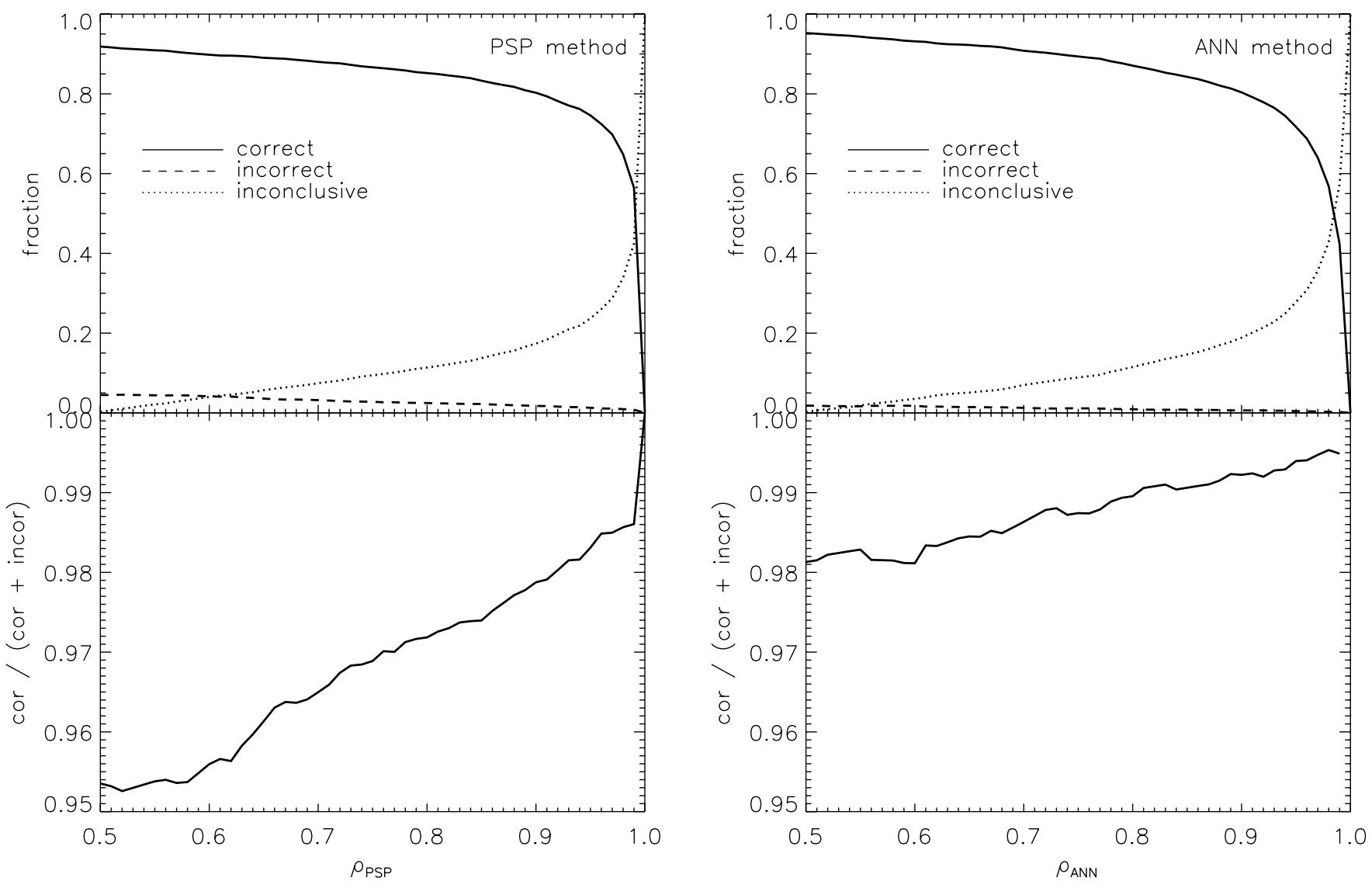

FIG. 3.-Top: Fraction of total evaluation-set galaxies correctly, incorrectly, and inconclusively identified in both methods vs. the accuracy parameter $\rho$. We allow the

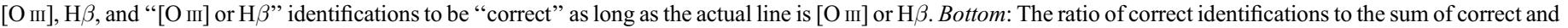
incorrect identifications, as defined in the top panel. [See the electronic edition of the Journal for a color version of this figure.]

each, shown diagrammatically in Figure 2. We find that other similar architectures do not affect line identification significantly.

In determining the functional relationship between inputs and outputs, an ANN finds the optimal configuration of weights to connect each node. Before training begins, the ANN is initialized with random weights. For this reason, ANNs with the same architecture and same training sets but with different initializations will usually find different local minima. The solution is to use the average of the results of a committee of separately initialized ANNs. We use committees of 20 ANNs identical in every way except for their initially randomized weights.

In analogy to the PSP method, we call the ANN outputs $P_{X}^{j}$, which are subjected to the seven conditions described in $\S 3$. Line $j$ is identified conclusively only if a single $P_{X}^{j}$ or $P_{\mathrm{O} \text { III }}^{j}+P_{\mathrm{H} \beta}^{j}$ exceeds $\rho_{\text {ANN }}$.

\section{ACCURACY}

Although setting aside evaluation sets reduces the training-set sizes and hence reduces precision, evaluation establishes confidence in the line identifications. In $\S 3.1$, we identified one line in each known-redshift galaxy to serve as the surrogate "single" line. In this section, we subject each evaluation galaxy to both methods and report the estimated identity of each line.

We choose $\rho_{\text {PSP }}=0.9$ and $\rho_{\text {ANN }}=0.8$, motivated by the arguments below. Tables 1-3 detail the results separated by field. Each column is an actual surrogate single line identity, and each row is a result from the identification algorithm. Correct identifications are shown in bold, where we consider the [O III], $\mathrm{H} \beta$, and " $[\mathrm{O}$ III $]$ or $\mathrm{H} \beta$ " categories to be correct for both $\left[\mathrm{O}_{\mathrm{III}}\right]$ and
$\mathrm{H} \beta$ lines. (If we assume the rest wavelength of the single line is the average of 4861 and $5007 \AA$, then the redshift will be skewed by $1.5 \%$, which is more precise than even the best photo- $z \mathrm{~s}$.)

The prior conditions in $\S 3$ improve the results significantly. The priors corrected 465 identifications in the PSP method, mostly [O III] and [O II], and 597 identifications in the ANN method,

TABLE 5

Single-Line Identification

\begin{tabular}{|c|c|c|c|}
\hline Identified as & Field 1 & Fields $2-4$ & All Fields \\
\hline \multicolumn{4}{|c|}{ PSP Method } \\
\hline $\mathrm{H} \alpha \ldots \ldots \ldots \ldots$ & 51 & 60 & 111 \\
\hline$[\mathrm{O}$ пII] $] \ldots \ldots \ldots \ldots \ldots \ldots \ldots$ & 16 & 16 & 32 \\
\hline $\mathrm{H} \beta$ & 12 & 13 & 25 \\
\hline 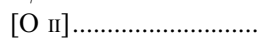 & 9 & 50 & 59 \\
\hline$[\mathrm{O}$ III] or $\mathrm{H} \beta \ldots \ldots \ldots \ldots$ & 85 & 89 & 174 \\
\hline 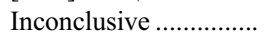 & 91 & 148 & 239 \\
\hline Total ............................. & 264 & 376 & 640 \\
\hline \multicolumn{4}{|c|}{ ANN Method } \\
\hline $\mathrm{H} \alpha$ & 79 & 87 & 166 \\
\hline$[\mathrm{O}$ III] & 55 & 47 & 102 \\
\hline $\mathrm{H} \beta \ldots \ldots \ldots \ldots \ldots \ldots$ & 4 & 11 & 15 \\
\hline 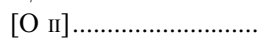 & 21 & 73 & 94 \\
\hline$[\mathrm{O}$ III] or $\mathrm{H} \beta \ldots \ldots \ldots \ldots \ldots$ & 40 & 55 & 95 \\
\hline 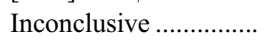 & 65 & 103 & 168 \\
\hline Total ….......................... & 264 & 376 & 640 \\
\hline
\end{tabular}



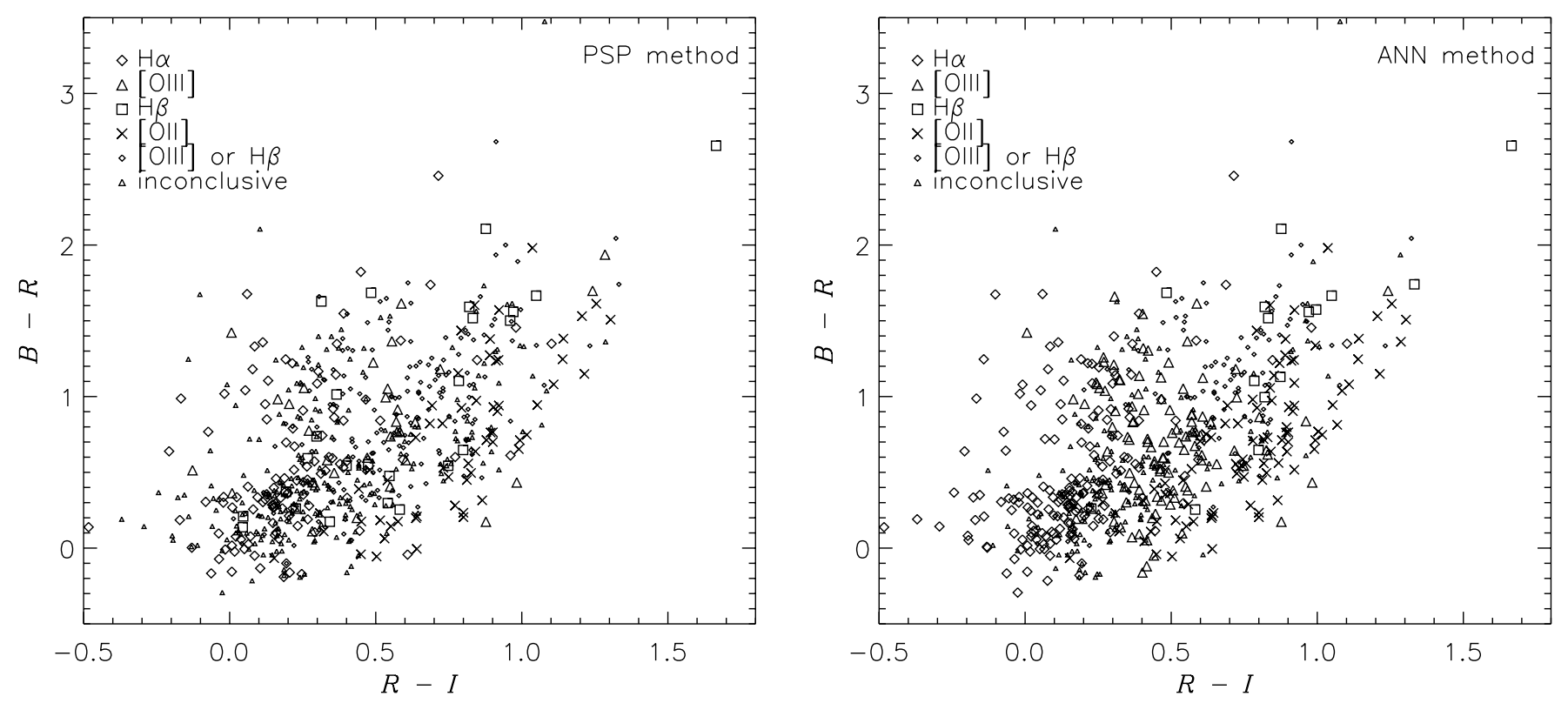

FIG. 4. - BRI color-color diagrams for the 640 single-line galaxies in all four fields color-coded by their identifications in both methods. Points that represent the "[O III] or $\mathrm{H} \beta$ " and "inconclusive" identifications are smaller so that they do not obscure the other points. [See the electronic edition of the Journal for a color version of this figure.]

overwhelmingly [O III]. The large majority of the identifications corrected to $\mathrm{H} \alpha$ and [O II] were inconclusive before the application of the prior conditions. Those corrected to [O III] were mostly "[O III] or $\mathrm{H} \beta$ " or inconclusive, and those corrected to $\mathrm{H} \beta$ were mostly " $[\mathrm{O}$ III] or $\mathrm{H} \beta$." The prior conditions work for many [O III] lines in the ANN method because the $P_{\mathrm{O} \text { III }}$ values for those lines are high even before the application of the prior conditions. In the PSP method, even though the prior conditions can eliminate $\mathrm{H} \beta$ as a possibility, $P_{\mathrm{O} \text { III }}$ is not large enough for a conclusive identification. Our choice of a smaller $\rho_{\text {ANN }}$ than $\rho_{\text {PSP }}$ creates this particular success of the ANN method.

Both methods show no large statistical difference between the fields with different selection functions. Field 1 contains more actual $\mathrm{H} \alpha$ lines than fields $2-4$, but the accuracy with which they are identified is about the same. This similarity between the fields justifies using one known-redshift set instead of one for field 1 and a different one for fields $2-4$.

The ANN method is superior to the PSP method in both accuracy and number of conclusive identifications. The PSP method in particular identifies $9.1 \%$ of the actual $\mathrm{H} \alpha$ lines as "[O $\mathrm{III}]$ or $\mathrm{H} \beta$ " compared to only $2.6 \%$ for the ANN method. Interestingly, $8.6 \%$ and $5.8 \%$ of the lines identified as $\mathrm{H} \alpha$ in the PSP and ANN methods are incorrect, despite the underrepresentation of $\mathrm{H} \alpha$ lines in fields 2-4. Not surprisingly, the PSP method performs poorly at identifying $[\mathrm{O}$ III] and $\mathrm{H} \beta$. However, the ANN method performs

TABLE 6

Comparison between PSP and ANN Methods on Single-Line Galaxies

\begin{tabular}{|c|c|c|c|c|c|c|c|}
\hline \multirow[b]{2}{*}{ PSP МЕtноD } & \multicolumn{6}{|c|}{ ANN Method } & \multirow[b]{2}{*}{ TотAL } \\
\hline & $\mathrm{H} \alpha$ & {$[\mathrm{O} \quad \mathrm{III}]$} & $\mathrm{H} \beta$ & [O пा] & {$[\mathrm{O} \mathrm{III}] / \mathrm{H} \beta$} & Inconclusive & \\
\hline $\mathrm{H} \alpha \ldots \ldots \ldots \ldots \ldots \ldots$ & 100 & 0 & 0 & 0 & 0 & 11 & 111 \\
\hline$[\mathrm{O}$ III] & 1 & 22 & 0 & 0 & 0 & 9 & 32 \\
\hline $\mathrm{H} \beta$ & 3 & 1 & 11 & 0 & 0 & 10 & 25 \\
\hline$[\mathrm{O}$ II $] \ldots \ldots \ldots \ldots \ldots .$. & 0 & 0 & 0 & 58 & 0 & 1 & 59 \\
\hline$[\mathrm{O}$ III] $]$ or $\mathrm{H} \beta \ldots \ldots$ & 1 & 61 & 4 & 0 & 87 & 21 & 174 \\
\hline Inconclusive ....... & 61 & 18 & 0 & 36 & 8 & 116 & 239 \\
\hline Total ................. & 166 & 102 & 15 & 94 & 95 & 168 & 640 \\
\hline
\end{tabular}

very well at identifying [O III] (91.4\% correct identifications) because the prior conditions correct so many identifications to [O III]. Every line classified as [O II] in both methods is correct, largely because of condition 7 in $\S 3$. The "[O III] or $\mathrm{H} \beta$ " category is $94.6 \%$ and $95.7 \%$ accurate for the PSP and ANN methods, respectively, but the ANN method conclusively and correctly identifies many more lines as [O $\mathrm{II}]$.

Table 4 shows the number of lines from the evaluation set that were identified the same and differently between the two methods. The numbers of lines that fall into the same category in both methods are shown in bold. Very few conclusive identifications are different between the two methods.

We find the optimal accuracy parameter $\rho$ for each method by attempting to maximize accuracy while minimizing the number of inconclusive identifications. We classify accurate $\mathrm{H} \alpha$, [O II],

TABLE 7

RECOVERED REDSHIFTS

\begin{tabular}{|c|c|c|c|c|c|}
\hline \multirow[b]{2}{*}{ IDENTIFIED AS } & \multicolumn{4}{|c|}{ Actual Line Identity } & \multirow[b]{2}{*}{ TотAL } \\
\hline & $\mathrm{H} \alpha$ & {$[\mathrm{O}$ III] } & $\mathrm{H} \beta$ & {$[\mathrm{O}$ пा] } & \\
\hline \multicolumn{6}{|c|}{ PSP Method } \\
\hline $\mathrm{H} \alpha \ldots \ldots \ldots \ldots \ldots \ldots$ & 5 & 0 & 0 & 0 & 5 \\
\hline$[\mathrm{O}$ III] & 0 & 2 & $\mathbf{0}$ & 0 & 2 \\
\hline $\mathrm{H} \beta$ & 0 & $\mathbf{0}$ & $\mathbf{0}$ & 0 & 0 \\
\hline 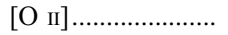 & 0 & 0 & 0 & 9 & 9 \\
\hline$[\mathrm{O}$ III $]$ or $\mathrm{H} \beta \ldots \ldots \ldots$ & 1 & 3 & 3 & 1 & 8 \\
\hline Inconclusive ........... & 2 & 5 & 0 & 2 & 9 \\
\hline Total ..................... & 8 & 10 & 3 & 12 & 33 \\
\hline \multicolumn{6}{|c|}{ ANN Method } \\
\hline $\mathrm{H} \alpha \ldots \ldots \ldots \ldots \ldots \ldots \ldots$ & 6 & 1 & 0 & 0 & 7 \\
\hline$[\mathrm{O}$ III] & 0 & 4 & $\mathbf{0}$ & 0 & 4 \\
\hline $\mathrm{H} \beta \ldots \ldots \ldots \ldots \ldots \ldots \ldots \ldots \ldots \ldots$ & 0 & $\mathbf{0}$ & $\mathbf{0}$ & 0 & 0 \\
\hline$[\mathrm{O}$ II $] \ldots \ldots \ldots \ldots \ldots \ldots$ & 0 & 0 & 0 & 10 & 10 \\
\hline$[\mathrm{O}$ III $]$ or $\mathrm{H} \beta \ldots \ldots \ldots$ & 0 & 2 & 3 & 0 & 5 \\
\hline Inconclusive .......... & 2 & 3 & 0 & 2 & 7 \\
\hline Total ....................... & 8 & 10 & 3 & 12 & 33 \\
\hline
\end{tabular}




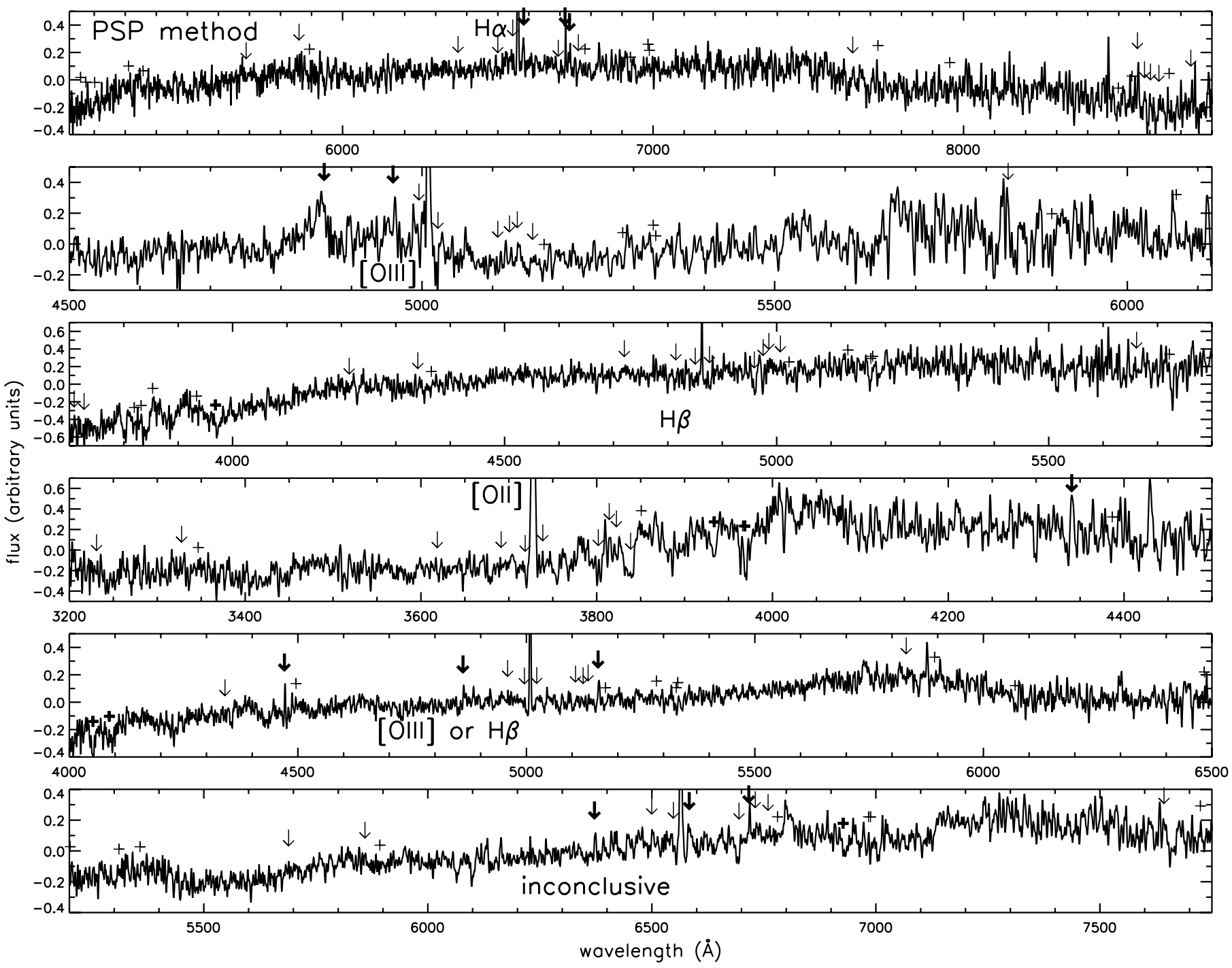

Fig. 5.-Co-added spectra of lines identified in each of the six categories. Each spectrum is labeled in angstroms and in the rest frame of the identified line. "[O III] or $\mathrm{H} \beta$ " is in the rest frame of $\left[\mathrm{O}_{\mathrm{III}}\right] \lambda 5007$, and "inconclusive" is in the rest frame of $\mathrm{H} \alpha \lambda 6563$. The symbols indicate spectral features at four different redshifts: red if the single line is $\mathrm{H} \alpha$, orange for [O $\mathrm{OII}]$, green for $\mathrm{H} \beta$, and blue for [ $\mathrm{O}$ II]. Arrows represent emission lines, and pluses represent absorption lines. Bold symbols draw attention to noticeable features, and thin symbols mark the locations of absent features. The peaks of the single lines are stronger than the maximum plotted flux. [See the electronic edition of the Journal for a color version of this figure.]

and "[O III] or $\mathrm{H} \beta$ " identifications as "correct." Individual [O III] and $\mathrm{H} \beta$ identifications are correct if the actual line is either [O III] or $\mathrm{H} \beta$. Lines where $P_{X}^{j}<\rho$ for all $X$ are "inconclusive," except where $P_{\mathrm{O} \text { III }}^{j}+P_{\mathrm{H} \beta}^{j}>\rho$. Inaccurate $\mathrm{H} \alpha$, [O II], and "[O III] or $\mathrm{H} \beta$ " identifications are "incorrect," as well as individual [O III] and $\mathrm{H} \beta$ identifications where the actual line is $\mathrm{H} \alpha$ or [O II]. Figure 3 shows the results for each method. We find that $\rho_{\mathrm{PSP}}=$ 0.9 and $\rho_{\text {ANN }}=0.8$ give ratios of correct to the sum of correct and incorrect results near 0.98 without sacrificing many conclusive identifications.

\section{RESULTS ON SINGLE-LINE GALAXIES}

\subsection{Single-Line Identification}

We apply both methods to the 640 truly single emission lines. Table 5 lists the fraction of single lines identified in each category separated by field. A comparison of each column in this table to the "Total" columns in Tables 1-3 immediately shows that the single-line population contains fewer [O II] lines because [O II] is often resolved and identifiable in DEEP2 spectra. It can be a single line only when the galaxy's internal velocities blend the doublet.

Of the four observables used to determine line identities, the two colors are the most powerful. Figure 4 shows the BRI colorcolor plane for the lines identified in both methods. It is useful to compare this figure with Figure 1. Conclusive line identifications are possible in regions where the training-set categories overlap because $R$ magnitude and observed wavelength also help to determine line identity and, more importantly, because the seven conditions described in $\S 3$ rule out certain line identities, leaving one dominant identity.

In contrast to the evaluation set, the priors change a large fraction of the single-line set identifications. In the PSP method, the priors changed the identifications of $517(80.8 \%)$ of the single lines, largely from $\mathrm{H} \beta$ to " $[\mathrm{O}$ III $]$ or $\mathrm{H} \beta$ " or from [O II $]$ to inconclusive. In the ANN method, priors changed the identifications of $285(44.5 \%)$ of the single lines, mostly from [O II] or inconclusive to $\mathrm{H} \alpha$, [O III], or $\mathrm{H} \beta$. While these large fractions may seem to diminish the power of the core PSP and ANN methods, the priors eliminate only one line in most cases. It is still up to the 


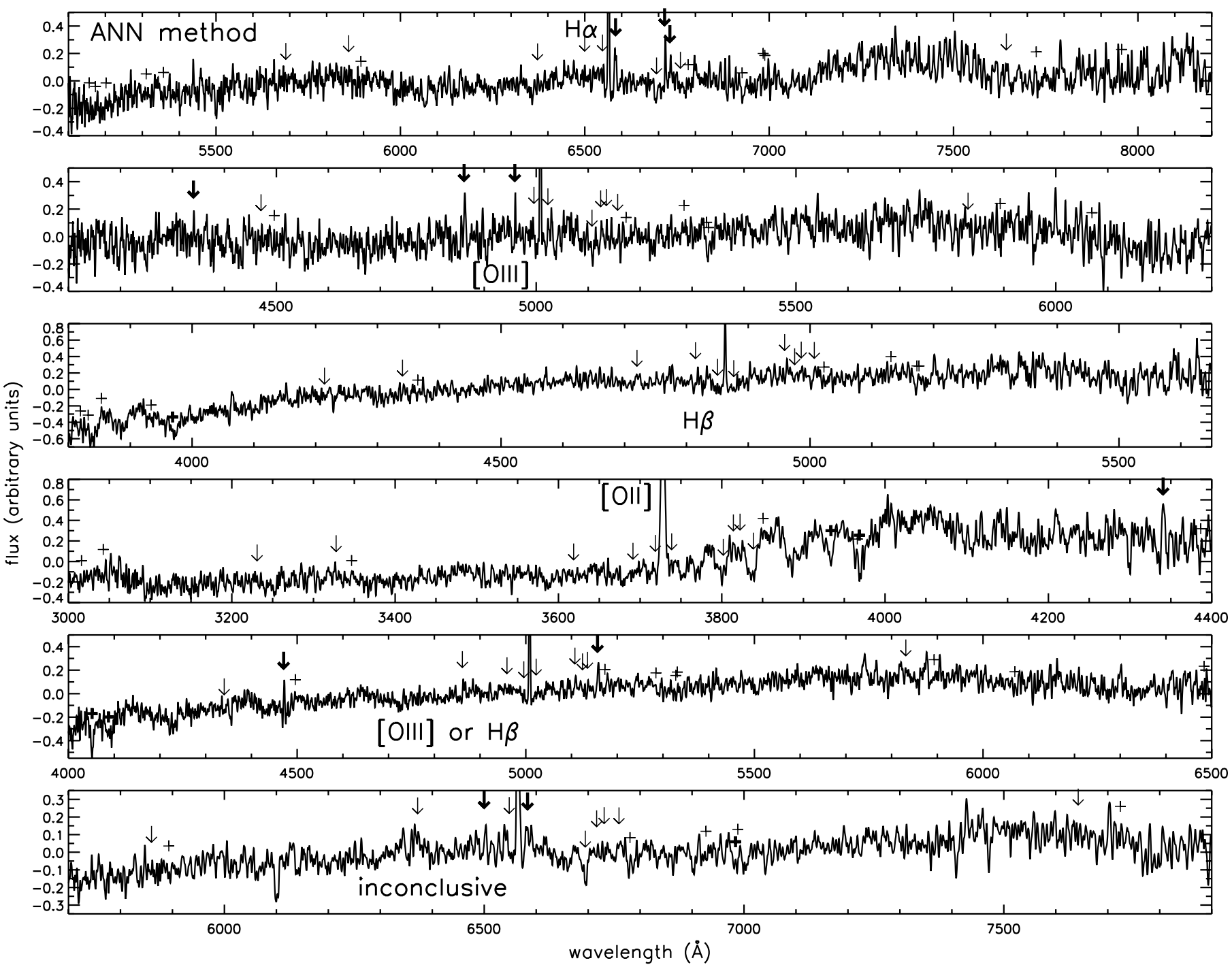

FIG. 5-Continued

core algorithm to choose among the remaining three lines based on broadband photometry and observed wavelength.

Table 6 shows the number of single lines that were identified the same and differently between the two methods. Conclusive line identifications do not change significantly between the two methods.

During the visual inspection of all single-line candidates, we identified the redshifts of 33 galaxies using additional lines that previous inspectors missed because they were dim or nearly hidden. Table 7 summarizes the results. The PSP method identifies 22 lines correctly, 2 incorrectly, and 9 inconclusively. The ANN method identifies 25 lines correctly, 1 incorrectly, and 7 inconclusively. Because these galaxies with recovered redshifts are so similar to the other single-line galaxies, their high success rate lends credence to both algorithms.

\subsection{Spectral Co-Addition}

A useful statistical check is to co-add the one-dimensional spectra of all of the galaxies identified in each category. We shift all the spectra to their rest frames and normalize them such that the median number of counts in a pixel is 1 . Then, we co-add them with inverse variance weighting. The co-added spectra are smoothed with a Gaussian window function of $\sigma=2.5$ pixels to approximate the instrumental resolution. If a line is identified properly, other spectral features associated with the single line should emerge above the noise. On the other hand, if the line is misidentified, some spectral features associated with other lines may be present. Although this technique cannot identify individual lines, it can give an idea of overall success or failure for each identification category.

Figure 5 shows the co-added spectra for the galaxies identified in each of the six categories. Emission and absorption features shown at four different redshifts - corresponding to the four allowed identities of each single line - are marked on each spectrum. The 14 features are [O II] $\lambda 3727, \mathrm{CaH} \lambda 3934, \mathrm{CaK} \lambda 3969$,

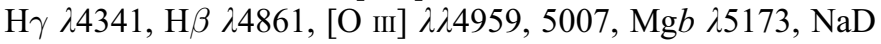
$\lambda 5893,[\mathrm{~N}$ II] $\lambda \lambda 6548,6583, \mathrm{H} \alpha \lambda$ 26563, and [S II] $\lambda \lambda 6716,6731$. Bold symbols direct attention to potentially real spectral features.

Both methods show [N II] and [S II] in the $\mathrm{H} \alpha$ spectra; [O III] $\lambda 4959$ and $\mathrm{H} \beta$ in the [O III] spectra; $\mathrm{CaK}$ in the $\mathrm{H} \beta$ spectra; and $\mathrm{CaH}, \mathrm{CaK}$, and $\mathrm{H} \gamma$ as well as high-order Balmer absorption in the [O II] spectra. The [O II] lines are broad, as expected. The "[O III] or $\mathrm{H} \beta$ " spectra for both methods display $\mathrm{CaH}, \mathrm{CaK}, \mathrm{H} \gamma$, and [O III] $\lambda 5007$ associated with $\mathrm{H} \beta$. The PSP method spectrum may also contain [O III] $\lambda 4959$ associated with [O III] $\lambda 5007$. Finally, the inconclusive category contains few convincing lines, 
but features associated with $\mathrm{H} \alpha$ and [O III] may be present. The presence of expected lines and absence of others in conclusively identified single-line galaxy spectra strengthens credibility in the identification of single emission lines.

\section{CONCLUSIONS}

The methods presented here combine photometry, spectroscopy, and physically motivated arguments about line flux ratios to determine the identity of single emission lines in galaxy spectra. The resultant redshifts seem very accurate. The parameter space proximity and neural network methods identify $82.6 \%$ and $88.5 \%$ of the lines in the evaluation set, and for the neural network method, over $98 \%$ of those identifications are correct. The spectral resolution of DEIMOS makes both methods nearly perfect at identifying [O II], but they make more mistakes in identifying $\mathrm{H} \alpha(8.6 \%$ and $5.8 \%$ failure rates $)$. The methods identify [O III] and $\mathrm{H} \beta$ lines as one of the two with $6.0 \%$ and $3.6 \%$ failure rates. Even this ambiguous identification can give a redshift more precise than present state-of-the-art photo-zs. Remarkably, the neural network method correctly identifies well over half of the [O III] lines without this ambiguity. The parameter-space proximity method recovers redshifts for $62.7 \%$ of the 640 single-line galaxies, and the neural network method recovers redshifts for $73.8 \%$ of the sample. Overall, the neural network method seems superior in both accuracy and recovery rate.

Identifying single emission lines is important to future work in DEEP2. The survey contains about 1000 serendipitously detected galaxies ("serendips"), many of which display only one emission line. The line identification algorithms may be applied to these objects and double the number of identified single lines. (One con- cern is that the serendips will occupy a region of parameter space not populated by DEEP2 targets in the training set.) In addition, the wealth of data in field 1, or the EGS, can increase accuracy and reduce inconclusive identifications with supplemental broadband measurements or morphological parameters, especially angular sizes, from high-resolution images. EGS photo-zs calculated without spectroscopic information can also constrain redshifts enough to identify single lines. Furthermore, all four fields also contain more information than we use in this work. For example, surface brightness, angular size, and the galaxy-galaxy correlation function all contain information about redshift. DEEP2 catalogs these three observables, which may be implemented in both single-line identification methods. Finally, although many single-line galaxy spectra display very faint continuums, cross-correlations with the continuums of known-redshift galaxies or templates can reveal more redshift information. We plan to address these possibilities in future work.

We thank the referee for a helpful report. We also thank J. A. Newman for providing statistics on repeat observations, and we thank C. M. Pierce for carefully reading a draft of this paper. We acknowledge National Science Foundation grants AST 05-07483 and AST 05-07428. E. N. K. is supported by a NSF Graduate Research Fellowship. Data herein were obtained at the W. M. Keck Observatory, which is operated as a scientific partnership among the California Institute of Technology, the University of California, and NASA. The Observatory was made possible by the generous financial support of the W. M. Keck Foundation.
Brodwin, M., Lilly, S. J., Porciani, C., McCracken, H. J., Le Fèvre, O., Foucaud, S., Crampton, D., \& Mellier, Y. 2006, ApJS, 162, 20

Coe, D., Benítez, N., Sánchez, S. F., Jee, M., Bouwens, R., \& Ford, H. 2006, AJ, 132, 926

Cohen, J. G., Hogg, D. W., Pahre, M. A., Blandford, R., Shopbell, P. L., \& Richberg, K. 1999, ApJS, 120, 171

Coil, A. L., Newman, J. A., Kaiser, N., Davis, M., Ma, C.-P., Kocevski, D. D., \& Koo, D. C. 2004, ApJ, 617, 765

Collister, A. A., \& Lahav, O. 2004, PASP, 116, 345

Cooper, M. C., Newman, J. A., Madgwick, D. S., Gerke, B. F., Yan, R., \& Davis, M. 2005, ApJ, 634, 833

Davis, M., et al. 2003, Proc. SPIE, 4834, 161

\section{REFERENCES}

Davis, M., et al. 2005, preprint (astro-ph/0507555)

Faber, S. M., et al. 2003, Proc. SPIE, 4841, 1657

Firth, A. E., Lahav, O., \& Somerville, R. S. 2003, MNRAS, 339, 1195

Lilly, S. J., Hammer, F., Le Fèvre, O., \& Crampton, D. 1995, ApJ, 455, 75

Osterbrock, D. E., \& Ferland, G. J. 2006, Astrophysics of Gaseous Nebulae and Active Galactic Nuclei (2nd ed.; Sausalito: University Science Books)

Storrie-Lombardi, M. C., Lahav, O., Sodre, L., Jr., \& Storrie-Lombardi, L. J. 1992, MNRAS, 259, 8P

Suchkov, A. A., Hanisch, R. J., \& Margon, B. 2005, AJ, 130, 2439

Vanzella, E., et al. 2004, A\&A, 423, 761

Way, M. J., \& Srivastava, A. N. 2006, ApJ, 647, 102 